Research Paper

\title{
Circular RNA ZNF609 promotes the malignant progression of glioma by regulating miR-1224-3p/PLK1 signaling
}

\author{
Senjie Du, Hongying Li, Fen Lu, Shang Zhang and Jian Tang ${ }^{\bowtie}$ \\ Department of Rehabilitation, Children's Hospital of Nanjing Medical University, Jiangsu, China. \\ $\triangle$ Corresponding author: Jian Tang, Department of Rehabilitation, Children's Hospital of Nanjing Medical University, No.72 Guangzhou Road, Gulou District, \\ Nanjing 210000, Jiangsu, China. Phone: +86- 025-83117558; E-mail: jiantang@njmu.edu.cn. \\ (C) The author(s). This is an open access article distributed under the terms of the Creative Commons Attribution License (https://creativecommons.org/licenses/by/4.0/). \\ See http://ivyspring.com/terms for full terms and conditions.
}

Received: 2020.10.24; Accepted: 2021.03.03; Published: 2021.04.12

\begin{abstract}
Objective: Previous studies have demonstrated that circular RNAs (circRNAs) play vital roles in pathological process of various diseases, including tumors. This study aimed at exploring the role and mechanism of circRNA RNA ZNF609 (circ-ZNF609) in the occurrence and development of glioma.

Materials and methods: Real-time quantitative PCR ( $q R T-P C R$ ) was applied to measure the expression of circ-ZNF609, miRNA-1224-3p (miR-1224-3p) and Polo-like kinase 1 (PLK1) in glioma tissues and cell lines. Furthermore, the association between circ-ZNF609 and clinical features of glioma was analyzed. CCK8 assay, EdU assay and Transwell assay were conducted to detect the effect of circ-ZNF609, miR-1224-3p and PLK1 on proliferation, migration and invasion in glioma cells. Then, we investigated the underlying mechanism of circ-ZNF609 by bioinformatics analysis, luciferase reporter assay, RNA immunoprecipitation (RIP), qRT-PCR and western blotting assay.

Results: Circ-ZNF609 was confirmed prominently upregulated in glioma. Inhibition of circ-ZNF609 could obviously suppress glioma cell proliferation, migration and invasion, while overexpression of circ-ZNF609 promoted glioma growth and metastasis. In vivo, xenotransplanted tumor model also showed that overexpression of circ-ZNF609 could promote in vivo glioma growth. Mechanistically, circ-ZNF609 could promote PLK1 expression via binding to miR-1224-3p, circ-ZNF609/miR-1224-3p/ PLK1 was shown responsible for circ-ZNF609 promoting glioma growth and metastasis.

Conclusion: Together, our results revealed that circ-ZNF609 elevates glioma growth and metastasis via enforcing PLK 1 expression by competitively binding miR-1224-3p, suggesting that circ-ZNF609 might be an underlying therapeutic target for glioma.
\end{abstract}

Key words: circular RNA ZNF609; glioma; miRNA sponge; malignant progression

\section{Introduction}

Glioma is the most prevalent type of primary nervous system tumors with a poor prognosis [1-3]. Glioblastoma is the most aggressive type of glioma and the median overall survival of it is within 15 months. Incidence and mortality of glioma appear to be increasing rapidly in the past few decades as a result of this highly metastatic disease $[4,5]$. Although the recent treatments like targeted biologic therapy and adjuvant radiotherapy have been markedly improved, overall patient survival is not satisfactory as a result of local recurrence and distant metastasis
$[6,7]$. Thus, identification of potential pathogenic mechanisms of glioma and therapeutic approaches against glioma are significantly indispensable.

Circular RNAs (circRNAs) is a novel member of noncoding RNAs (ncRNAs). Different from linear RNAs, circRNAs are characterized by free $5^{\prime}$ - or 3 '-ends connection with more stable structure $[8,9]$. Although advanced achievements have been made in recent years, the underlying regulatory mechanism of many circRNAs remain to be further explored. Moreover, circRNAs have been regarded as a 
"molecular sponge" to promote the expression of the downstream genes via harboring multiple microRNAs (miRNAs) and affect their activity therefore [10, 11]. Emerging evidence demonstrates that circRNAs have attracted more attention in various diseases by regulating many pathological and physiological process [12-14], including cell growth, cell cycle, apoptosis, metastasis and differentiation. For examples, circRNA hsa_circ_0072995 promoted the progression of epithelial ovarian cancer (EOC) via modulating miR-147a/CDK6 axis[15]; has_circ_0000140 could restrain the cell proliferation, migration, invasion and glycolysis metabolism of oral squamous cell carcinoma (OSCC) by promoting CDC73 expression via binding to miR-182-5p[16]; circRNA hsa_circ_0002577 has been verified to be upregulated in endometrial cancer and it might accelerate cancer progression by activating IGF1R/ PI3K/Akt pathway [17]. However, the potential biological roles of circRNAs in glioma remain to be further explored.

Circ-ZNF609 (circBase ID: hsa_circ_0000615) is located in chromosome $15.64 \mathrm{q}$ and originated from ZNF609 [18]. ZNF609 is a member of zinc finger family, which is involved in regulation of gene expression [19]. CircZNF609 has been reported to be dysregulated in many tumors such as renal carcinoma [20], lung adenocarcinoma [21], nasopharyngeal carcinoma [22], colorectal cancer [23], hepatocellular carcinoma [24], gastric cancer [25] and prostate cancer [26]. Nevertheless, the biological roles of circZNF609 in glioma remain unclear. In this study, we verified a circRNA deriving from the ZNF609 gene locus, named circ-ZNF609 whose expression was increased in glioma. Besides, in vitro and in vivo assays demonstrated that circ-ZNF609 was positively interrelated to the growth and metastasis of glioma. Mechanistically, circ-ZNF609 might promote PLK1 expression by binding to miR-1224-3p, so as to promote glioma growth and metastasis. Hence, circ-ZNF609 might be a potential clinical biomarker and therapeutic target in glioma.

\section{Material and Methods}

\section{GEPIA database}

GEPIA is a new interactive website for TCGAand GTEx-based analysis of RNA sequence data (http://gepiacancer-pku.cn). A total of 518 glioma tissues and 207 normal control tissues were obtained from TCGA database to analyze mRNA expression profile. ZNF609 expression in low grade glioma tissues and normal control tissues were analyzed.

\section{Clinical samples}

62 glioma tissues and 15 non-glioma tissues were recruited from the Children's Hospital of Nanjing Medical University between January 2015 and October 2018. None of the patients had preoperative radiotherapy or chemotherapy. All the tissues were collected by surgery and confirmed by two independent pathologists. The tissue biopsies were stored at $-80{ }^{\circ} \mathrm{C}$. The informed consent forms were obtained from all participants and this study was approved by the Medical Ethics Committee of Children's Hospital of Nanjing Medical University.

\section{Cell culture and transfection}

The glioma cell lines (LN229, LN308, U87 and U251) and HEB (human normal brain glial cells) were obtained from the Chinese Academy of Sciences. All the cells were cultivated in Dulbecco's modified Eagle's Medium (Gibco, USA) containing 10\% fetal bovine serum (Gibco, USA) at $37^{\circ} \mathrm{C}$ with $5 \% \mathrm{CO}_{2}$.

For circ-ZNF609 overexpression, circ-ZNF609 overexpression plasmids were constructed into pSicoR lentiviral vector, and 293T cells were used for virus production. Lentiviruses were used to infect glioma cells and puromycin was used to select for two weeks to produce circ-ZNF609 overexpressed cell lines. U87 and U251 cells were placed in 6-well plates (5x104 per well) and transfected with circ-ZNF609 siRNAs, miR-1224-3p mimics and inhibitor, PLK1 siRNAs and corresponding negative references by using Lipofectamine 3000 (Invitrogen, USA) for $24 \mathrm{~h}$ in accordance with the manufacturer's protocol. All the transfection reagents were generated by GenePharma (Shanghai, China).

\section{Quantitative real-time polymerase chain reaction (qRT-PCR)}

Total RNA from glioma tissues and cell lines was extracted by utilizing TRIzol reagent (Invitrogen, USA) in accordance with the manufacturer's instructions. Then, reverse transcription was conducted to obtain cDNA. Subsequently, the qRT-PCR assay was conducted with SYBR Green PCR Master Mix (TakaRa, Japan) on LightCycler system (Roche, Basel, Switzerland). RNase R assay and Actinomycin D assay were utilized to verify the stability of circ-ZNF609 and linear ZNF609 mRNA expression level. The relative expression level was examined by the 2- $\triangle \triangle \mathrm{CT}$ method and GAPDH or U6 small nuclear RNA was used as internal controls. The primer sequences are shown in Table 1.

\section{Cell proliferation assay}

CCK8 assay: After transfection, cells $\left(4 \times 10^{3}\right)$ were transferred into a 96-well plate. After incubation for $24 \mathrm{~h}$, each well was added with $10 \mu \mathrm{l}$ of CCK-8 reagent for $2 \mathrm{~h}$ at $37^{\circ} \mathrm{C}$, after the indicated time $(0,24$, 
48,72 and $96 \mathrm{~h}$ ). Then, the OD values at $450 \mathrm{~nm}$ were examined by a microplate reader at $450 \mathrm{~nm}$.

Table 1. Sequences of primers for qRT-PCR

\begin{tabular}{|c|c|}
\hline Name & Sequence \\
\hline \multicolumn{2}{|l|}{ Circ-ZNF609 } \\
\hline Forward & $5^{\prime}$ - TGAGTGTCGCCTGCTAAAGA-3' \\
\hline Reverse & 5' - СССССАGСТTTCСТАТTTTC-3' \\
\hline \multicolumn{2}{|l|}{ miR-1224-3p } \\
\hline Forward & 5'- GAGGACTCGGGAGGTGGAG-3' \\
\hline Reverse & 5'- CTGAGGAGAGAGGAGGTGGG-3' \\
\hline \multicolumn{2}{|r|}{ - the } \\
\hline Forward & 5'- AAAGAGATCCCGGAGGTCCTA-3' \\
\hline Reverse & 5'- GGCTGCGGTGAATGGATATTTC-3' \\
\hline \multicolumn{2}{|r|}{ 200 } \\
\hline Forward & 5'- TGCACCACCAACTGCTTAGC-3' \\
\hline Reverse & 5'- GGCATGGACTGTGGTCATGAG-3' \\
\hline \multicolumn{2}{|r|}{ 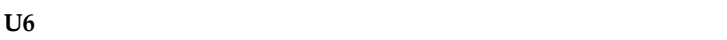 } \\
\hline Forward & 5'-CGCTTCGGCAGCACATATAC-3' \\
\hline Reverse & 5'- TTCACGAATTTGCGTGTCAT-3' \\
\hline
\end{tabular}

EdU assay: EdU kit (RiboBio, China) was utilized for the assay. Transfected glioma cells were placed in 96-well plate at 3000 cells/well, and then the culture medium was added with $50 \mathrm{mM}$ EdU solution. $24 \mathrm{~h}$ later, cells were fixed in $4 \%$ formaldehyde and infiltrated using Triton X-100. Treated cells were incubated with EdU reagent and counterstained using Hoechst (Thermo Fisher, USA). The staining results under fluorescence microscope were calculated. Pictures of five fields of view randomly selected under microscope were taken to further analyze.

\section{Transwell assay}

Transwell assay was conducted using 24-well plate with membrane filter chamber at aperture $8 \mu \mathrm{m}$. For invasion assay, Matrigel ${ }^{\mathrm{TM}}$ (BD Biosciences) was precoated in the upper chamber. U87 and U251 cells were selected into the upper chamber at $5 \times 10^{4} /$ well by using $100 \mu \mathrm{L}$ FBS-free DMEM culture medium, while $600 \mu \mathrm{l}$ DMEM culture medium with $10 \%$ FBS were seeded into the lower chamber. The chambers were incubated at $37^{\circ} \mathrm{C}$ for $24 \mathrm{~h}$, the cells in upper chamber were removed by cotton swabs. Then the cells adhering to the under layer of the membranes were fixed into methanol and stained using crystal violet. Finally, a microscope was used to capture the images of cells. Transwell plates used for the migration assay did not have the upper chambers coated with Matrigel.

\section{Subcellular fractionation location}

Briefly, we transferred glioma cells into EP tubes and added Lysis Buffer J for $20 \mathrm{~min}$. After centrifugation, the obtained supernatant contained cytoplasmic RNA. Next, we incubated the remaining liquid with nuclear lysate solution (RIPA) for $20 \mathrm{~min}$. After centrifugation, the obtained supernatant contained nuclear RNA. QRT-PCR assay was performed to verify the subcellular localization of circ-ZNF609.

\section{Luciferase assay}

Circ-ZNF609 and PLK1 3'untranslated region ( $\left.3^{\prime} \mathrm{UTR}\right)$ wild sequence and mutant sequence with or without miR-1224-3p binding sites were synthesized by Genepharm. According to the instructions of the manufacturer, Promega kit (Promega, USA) was employed for measuring luciferase activity as per the IFU and recorded.

\section{RNA immunoprecipitation}

U87 or U251 cells were collected and lysed using Magna RIP Kit (EMD Millipore), and then incubated with protein G Sepharose beads (GE Healthcare) coated with anti-AGO2 antibody (Abcam) at $4{ }^{\circ} \mathrm{C}$ overnight, and anti-IgG antibody was used as the negative control. RNA was then isolated for qRT-PCR.

\section{Western blot}

The proteins were extracted from glioma tissues or cell lines utilizing lysis buffer of protease inhibitor PMSF. The BCA Kit was applied to analyze the protein concentration. Then proteins $(20 \mu \mathrm{g})$ were separated on SDS-PAGE gels and blotted on PVDF membrane. After blocking in 5\% skim milk, the primary antibody was added for incubation. After cleaning the membrane, secondary antibody was incubated before exposure. Then the membrane incubated with primary antibodies targeting PLK1 (ab189139; Abcam, Cambridge, MA, USA) or GAPDH (1:1000; Beyotime, Nantong, China) overnight at $4{ }^{\circ} \mathrm{C}$. Next, the membranes were washed using TBS for three times and incubated with an HRP-linked goat antirabbit secondary antibody (ab205718; Abcam) at room temperature for $2 \mathrm{~h}$. The ECL Western Blotting Substrate Kit (ab65623; Abcam) was used to detect protein signals.

\section{Xenograft mouse models}

After LV-circ-ZNF609-U87 cells and LV-NC-U87 cells were stably transfected, approximately $2 \times 10^{5}$ stably transfected cells were injected subcutaneously into the right limbs of the e BALB/c nude mice. Tumor weights and volumes (volumes $\left(\mathrm{mm}^{3}\right)=$ length $\times$ width $^{2} / 2$ ) were measured 30 days after subcutaneous injection. This study had gained approval of the ethics committee of Children's Hospital of Nanjing Medical University. 


\section{Statistics analysis}

All the results were expressed as mean \pm SEM and analyzed with GraphPad Prism 8.0 software. Student's t-test was utilized for the comparison of between-group difference. The overall survival rate (OS) was analyzed by Kaplan-Meier method and the log-rank test. All the assays were repeated for three times. $\mathrm{P}<0.05$ was considered statistically significant.

\section{Results}

\section{Circ-ZNF609 expression level was obviously elevated in glioma}

Firstly, we detected the expression of circ-ZNF609 in glioma tissues and normal control tissues by conducting qRT-PCR assay. The results indicated that the level of circ-ZNF609 in glioma was markedly increased. Besides, the expression level of circ-ZNF609 in high grade glioma was higher than that in low grade glioma (Figure 1A). We measured the expression of circ-ZNF609 in glioma cell lines, and found that circ-ZNF609 expression was markedly increased in glioma cell lines compared to HEB (Figure 1B). After analyzing, we found that circ-ZNF609 expression was interrelated to the poor prognosis of glioma patients (Figure 1C). Furthermore, the results of GEPIA database indicated that linear ZNF609 was much higher in low grade glioma tissues than that in normal tissues (Figure 1D). Besides, to examine the stability of circ-ZNF609, the transcriptional inhibitor actinomycin D was used to co-culture with U87 and U251 cells and then detected the expression of circ-ZNF609 and linear ZNF609. The results indicated that the half-life of linear ZNF609 mRNA was less than $12 \mathrm{~h}$, while that of circ-ZNF609 was more than $24 \mathrm{~h}$ (Figure 1E). Moreover, the results of RNase $\mathrm{R}$ experiment indicated that RNase $\mathrm{R}$ treatment could significantly reduce the mRNA expression level of ZNF609, while the expression level of circ-ZNF609 was not affected after RNase R treatment (Figure 1F).
A

D
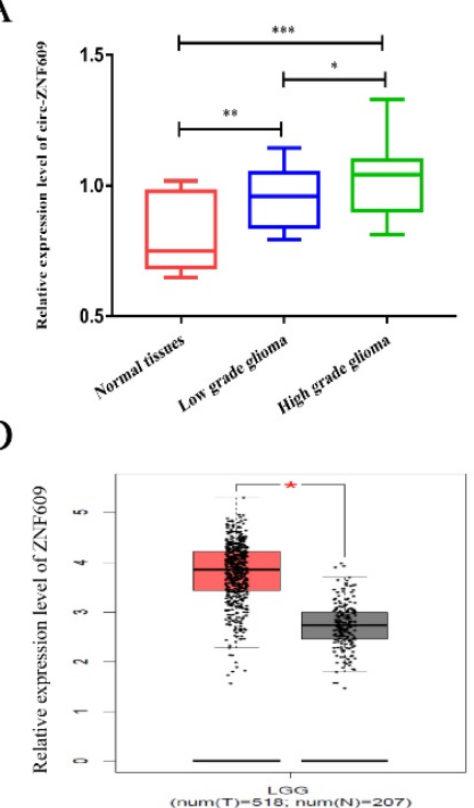

$\mathrm{B}$

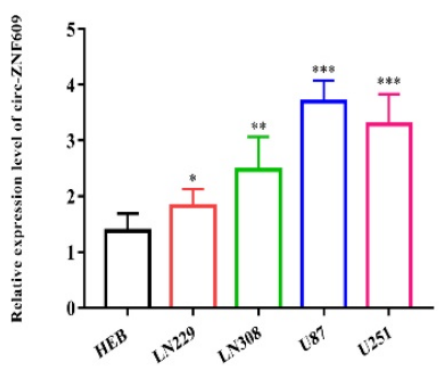

E

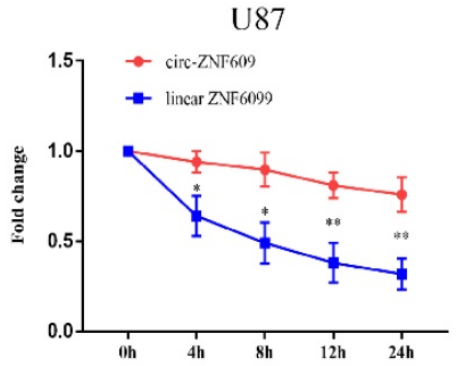

C

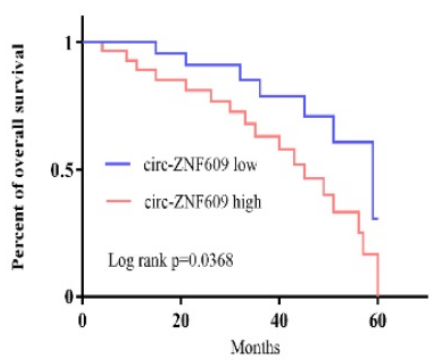

$\mathrm{U} 251$

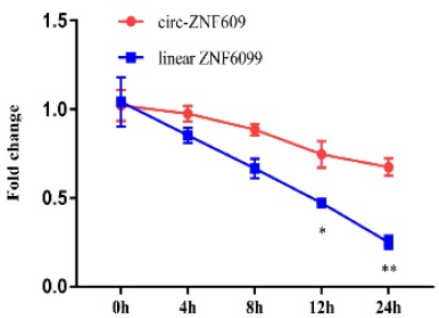

$\mathrm{F}$

U87

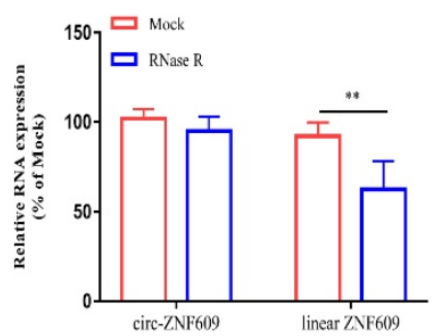

$\mathrm{U} 251$

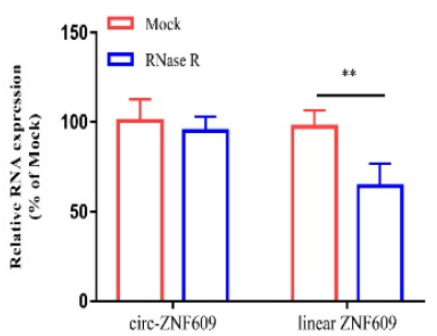

Figure 1. Circ-ZNF609 expression level was obviously elevated in glioma. (A) The expression level of circ-ZNF609 in glioma tissues was increased compared to expression in matched normal tissues. (B) Relative expression of circ-ZNF609 in glioma cell lines compared to expression in HEB cells. (C) Kaplan-Meier analysis of the overall survival of glioma patients with high and low expression of circ-ZNF609 ( $p=0.0368)$. (D) ZNF609 expression in low grade glioma tissues was analyzed by TCGA database. (E) U87 and $U 251$ cells were treated with actinomycin D, the mRNA expression of circ-ZNF609 and ZNF609 was measured by qRT-PCR. (F) The stability of circ-ZNF609 was evaluated by RNase R test in U87 and U251 cells. ${ }^{*} \mathrm{p}<0.05$; ${ }^{*} \mathrm{p}<0.01$; ${ }^{* *} \mathrm{p}<0.001$. All data are showed as mean $\pm \mathrm{SD}$. The experiment was conducted for three times. 


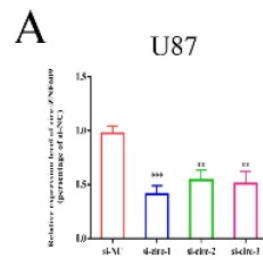

D $\quad$ si-NC

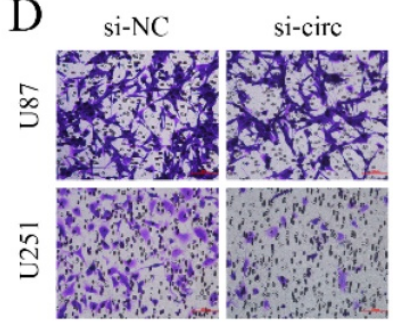

B

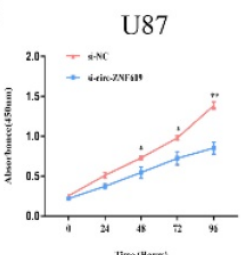

(ime: Haren)

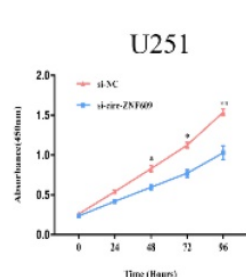

thise (haten)

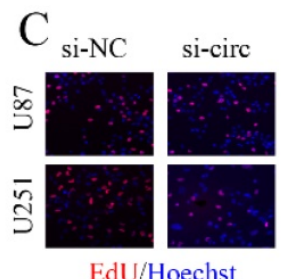

EdU/Hoechst

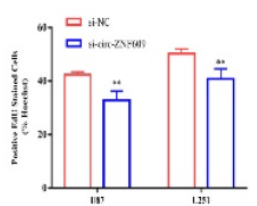

si-circ
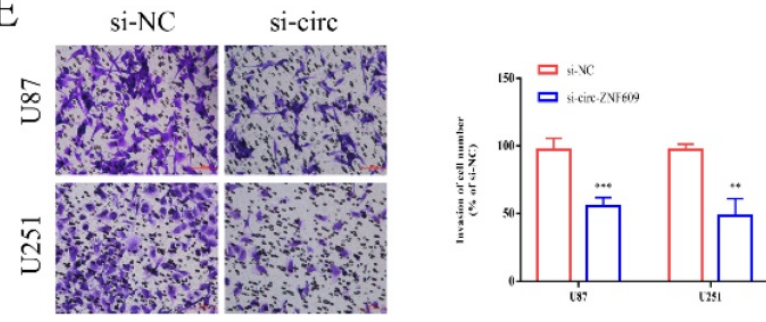

Figure 2. Inhibition of circ-ZNF609 suppressed the proliferation, migration and invasion of glioma cells. (A) Specific small interference RNA (siRNAs) was synthesized in accordance with circ-ZNF609 sequence and transfected into U87 and U251 cells. The interference efficiency was detected by qRT-PCR. (B-C) CCK8 assay and EdU assay were applied to measure the effect of circ-ZNF609 on the proliferation of U87 and U251 cells (magnification: 200x). The results showed that inhibition of circ-ZNF609 could suppress the proliferation of glioma cells. (D-E) The metastatic ability of U87 and U251 cells was examined by transwell migration and invasion assay after suppressing the expression of circ-ZNF609 in U87 and U251 cell lines (magnification: 200x). The results showed that inhibition of circ-ZNF609 could suppress the migration and invasion of glioma cells. ${ }^{*}<<0.05 ;{ }^{* *} p<0.01 ;{ }^{* * *} p<0.001$. All data are showed as mean \pm SD. The experiment was conducted for three times.

\section{Inhibition of circ-ZNF609 suppressed the proliferation, migration and invasion of glioma cells}

To confirm the potential function of circ-ZNF609 in glioma, small interfering RNAs (siRNAs) were applied to interfere with the expression level of circ-ZNF609 in U87 and U251 cell lines. The results of qRT-PCR indicated that circ-ZNF609 siRNAs could effectively suppress the expression of circ-ZNF609, while the interference efficiency of siRNA-1 was higher than that of others (Figure 2A). Then, we detected the biological function of circ-ZNF609 on cell proliferation, migration and invasion in glioma cell lines. The results of CCK8 assay and EdU assay indicated that inhibition of circ-ZNF609 could markedly repress cell proliferation (Figure 2B-C). Moreover, transwell assay verified that knockdown of circ-ZNF609 could remarkedly suppress migration and invasion of glioma cell lines (Figure 2D-E). All above results proved that circ-ZNF609 could promote cell proliferation, migration and invasion in glioma.

\section{Circ-ZNF609 could bind to miR-1224-3p}

Emerging evidence has demonstrated that circRNAs could serve as ceRNAs and regulate downstream genes expression via sponging miRNAs. Hence, bioinformatics (https://circinteractome.nia. nih.gov/) was analyzed to detect the potential miRNAs might sponge circ-ZNF609, and miR-1224-3p possessed the highest bound fraction with circ-ZNF609 (Figure S1A). Then, we measured the expression of miR-1224-3p in glioma cell lines. The results indicated that the relative level of miR-1224-3p in glioma cell lines was significantly decreased
(Figure 3A). Then, we conducted nuclear-cytoplasmic separation assay and identified that circ-ZNF609 might exist mainly in the cytoplasm of U87 and U251 cells, suggesting that circ-ZNF609 could participate in post-transcriptional regulation. Therefore, miR-1224$3 p$ was chosen for further research. According to the predicted binding sites, we synthesized circ-ZNF609 wild-type plasmids (circ-wt) and mutant plasmids (circ-mut) (Figure S1B). Dual-luciferase reporter assay was performed to clarify the binding relationship between circ-ZNF609 and miR-1224-3p. As shown in Figure $3 \mathrm{C}$, the results of luciferase reporter assay demonstrated that miR-1224-3p mimics remarkedly suppressed the luciferase activity of circ-ZNF609-wt reporter, suggesting that miR-1224-3p might sponge circ-ZNF609. RNA immunoprecipitation (RIP) assay showed that both circ-ZNF609 and miR-1224-3p were significantly enriched in Ago2-containing beads compared with IgG-containing beads or the input group (Figure 3D) Moreover, qRT-PCR revealed that circ-ZNF609 inhibition promoted miR-1224-3p expression (Figure 3E). Additionally, qRT-PCR indicated that miR-1224-3p expression was obviously decreased in glioma tissues (Figure 3F). Besides, correlation analysis verified that circ-ZNF609 expression was negatively interrelated to $\mathrm{miR}-1224-3 p$ expression in low grade glioma tissues $\left(R^{2}=0.36\right.$, $\mathrm{p}<0.001$ ) (Figure $3 \mathrm{G}$ ) and high grade glioma tissues $\left(\mathrm{R}^{2}=0.32, \mathrm{p}<0.001\right)$ (Figure $\left.3 \mathrm{H}\right)$.

\section{MiR-1224-3p served as a suppressor in the proliferation, migration, and invasion of glioma cells}

To confirm the functional interaction between circ-ZNF609 and miR-1224-3p in glioma cells, we 
conducted the rescue experiment using qRT-PCR assay, CCK8 assay, EdU assay and transwell assay. As shown in Figure 4A, the miR-1224-3p inhibitor significantly decreased the expression level of miR-1224-3p, while co-transfection of circ-ZNF609-si promoted miR-1224-3p expression level. The results of CCK8 assay and EdU assay demonstrated that transfection of miR-1224-3p inhibitor remarkedly promoted the proliferation of U87 and U251 cells, while circ-ZNF609 overexpression plasmid (circZNF609-si) could relatively rescue the facilitating effect of miR-1224-3p inhibitor (Figure 4B, C). Furthermore, the results of transwell assay suggested that the inhibition in miR-1224-3p facilitated the migration and invasion of U87 and U251 cells, while inhibiting circ-ZNF609 expression level could reduce the facilitating effect of miR-1224-3p inhibitor (Figure $4 \mathrm{D}, \mathrm{E})$.

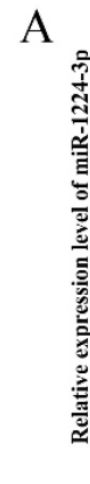

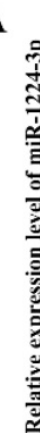

$\mathrm{C}$

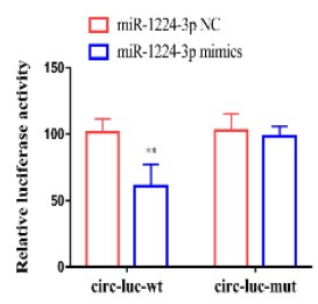

$\mathrm{U} 251$

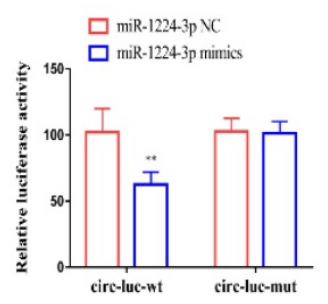

$\mathrm{F}$

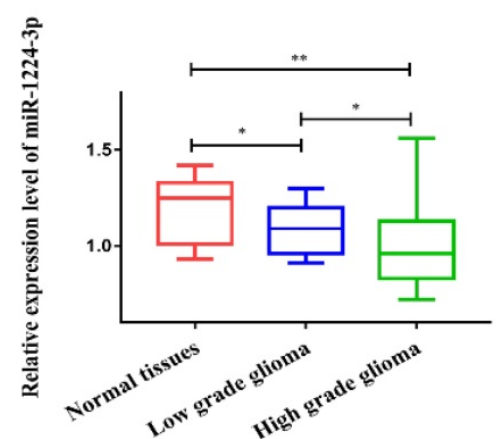

D
$\mathrm{B}$
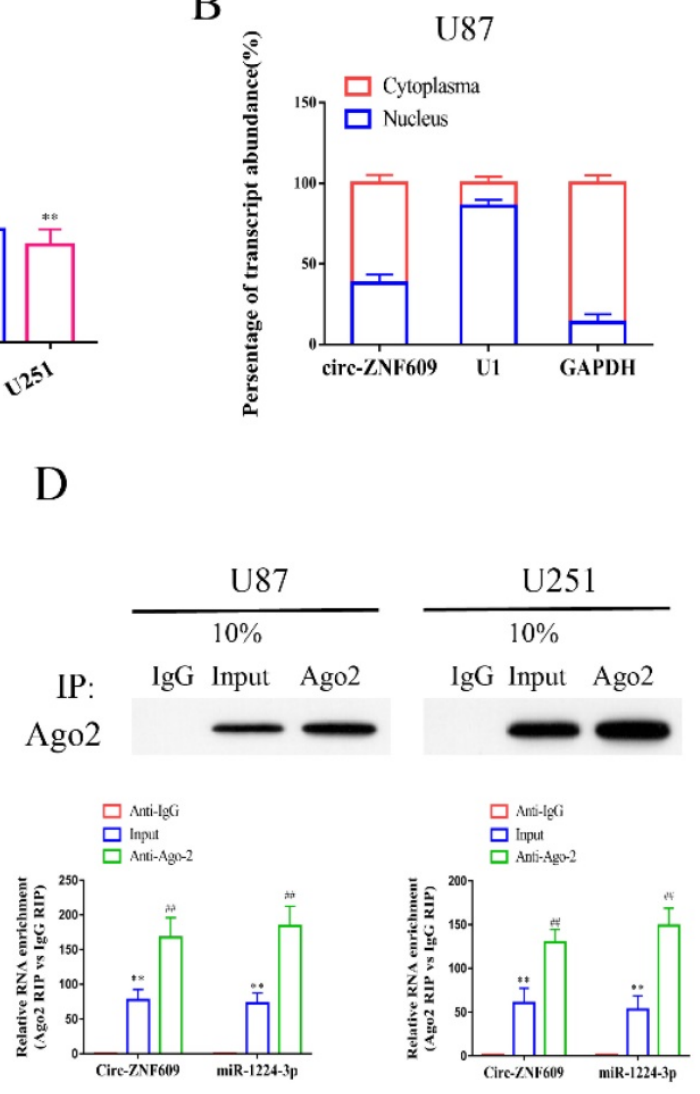

G

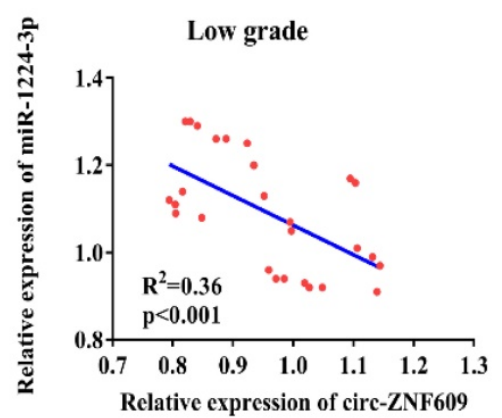

U251

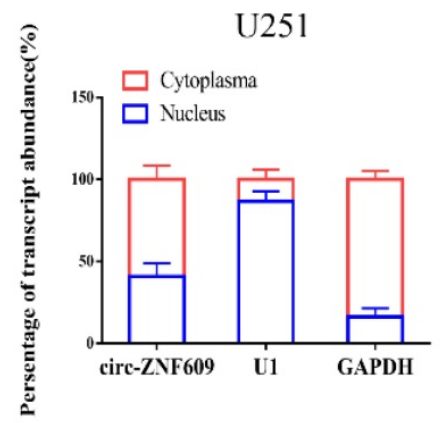

E

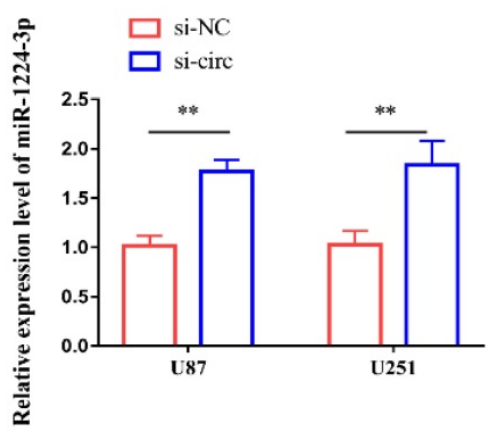

$\mathrm{H}$

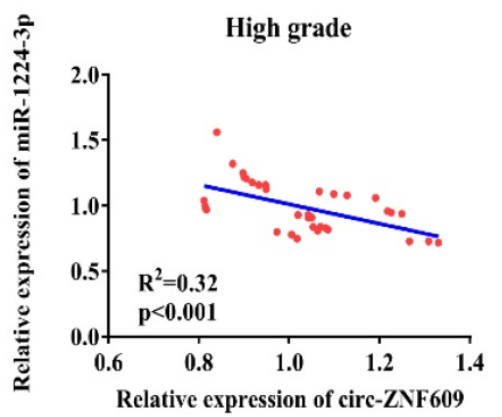

Figure 3. Circ-ZNF609 could bind to miR-1224-3p. (A) Relative expression of miR-1224-3p in glioma cell lines compared to expression in HEB cells. (B) Subcellular positioning of circ-ZNF609 in U87 and U251 cells through nuclear-cytoplasmic separation assay, the results showed that circ-ZNF609 is mainly located in the cytoplasm of glioma cell lines. (C) Relative luciferase activities were investigated in glioma cells after transfection with circ-ZNF609-WT or circ-ZNF609-Mut and miR-1224-3p mimic or miR-NC. (D) Enrichment of circ-ZNF609 and miR-1224-3p in Ago2-containing beads of U87 and U251 cells. (E) The expression of miR-1224-3p by qRT-PCR was detected after inhibition of circ-ZNF609 in U251 and U87 cells. (F) The expression of miR-1224-3p in glioma and adjacent tissues was tested by qRT-PCR, the results showed that the expression level of miR-1224-3p in glioma tissues was decreased compared to expression in matched normal tissues. (G-H) Through Person's correlation analysis, it was found that the expression of circ-ZNF6090 in low grade $\left(R^{2}=0.36, p<0.001\right)$ and high grade glioma tissue $\left(R^{2}=0.32, p<0.001\right)$ was significantly negatively correlated with mi $R-1224-3 p$ expression. ${ }^{*}<<0.05$; $*^{* *} \mathrm{p}<0.01 ;{ }^{*} \mathrm{p}<0.01$. All data are showed as mean $\pm \mathrm{SD}$. The experiment was conducted for three times. 
A
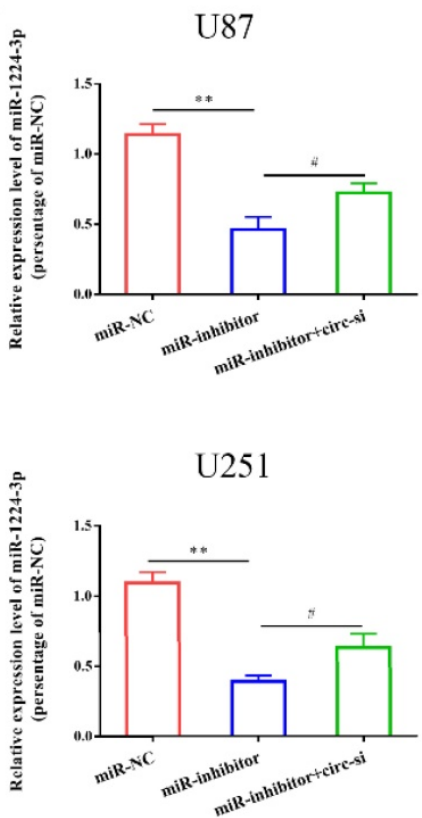

B
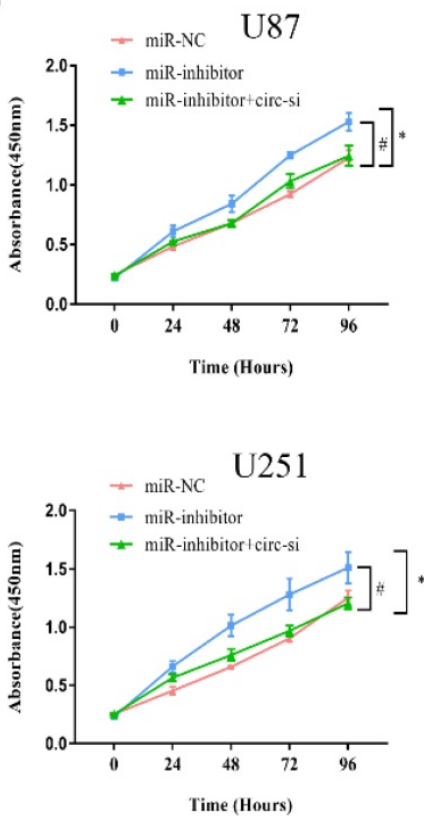

$\mathrm{C}$

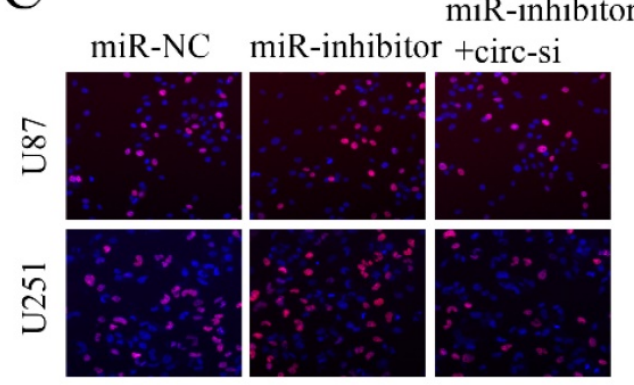

EdU/Hoechst

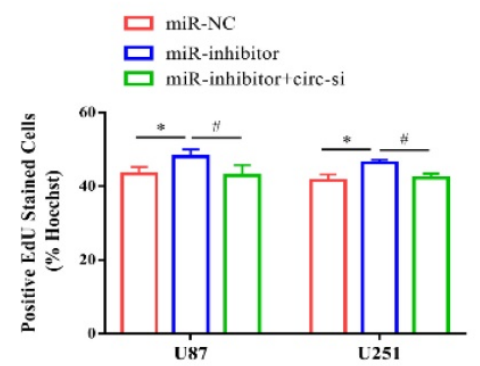

miR-inhibitor

miR-inhibitor

E
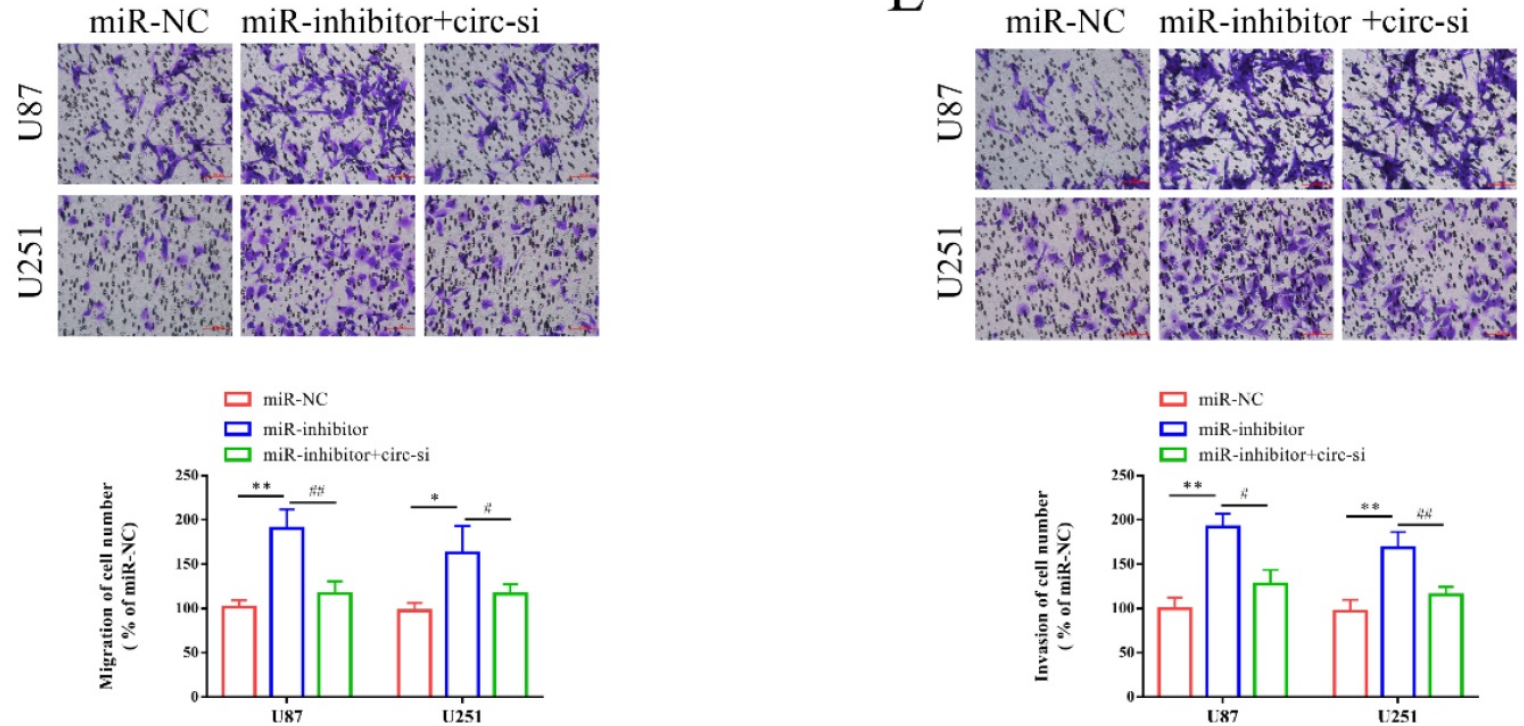

Figure 4. MiR-1224-3p served as a suppressor in the proliferation, migration, and invasion of glioma cells. (A) After inhibition of miR-1224-3p and circ-ZNF609 in U87 and U251 cells, the expression of miR-1224-3p was measured. (B-C) The proliferation of U87 and U251 cells were detected by CCK8 assay and EdU assay after suppressing miR-1224-3p and circ-ZNF609 simultaneously (magnification: 200x). (D-E) After inhibition of miR-1224-3p and circ-ZNF609 in U87 and U251 cells, the ability of cell migration and invasion was detected by Transwell assays (magnification: $200 \mathrm{x}$ ). ${ }^{*} \mathrm{p}<0.05 ;{ }^{* *} \mathrm{p}<0.01 ;{ }^{\#} \mathrm{p}<0.05 ;{ }^{\#} \mathrm{p}<0.01$. All data are showed as mean \pm SD. The experiment was conducted for three times.

\section{PLK1 is a target of miR-1224-3p}

Next, we explored the potential target genes of miR-1224-3p through RNAhybird, microRNA.org, TargetScan and miRDB, and we determined that PLK1 may be the downstream gene. Hence, we chose it for further study (Figure S1C). According to the predicted binding sites, we synthesized a PLK1 wild type plasmid (PLK1-wt) and a mutant plasmid (PLK1-mut) (Figure S1D). As shown in Figure 5A, the results of luciferase reporter assay showed that miR1224-3p mimics markedly suppressed the luciferase activity of PLK1-wt reporter, suggesting that miR1224-3p might target PLK1. Furthermore, we explored the expression level of PLK1 in glioma and found that PLK1 expression was extremely increased in glioma tissues and cell lines (Figure 5B,C). Then, we analyzed the correlation between miR-1224-3p and PLK1 in glioma tissues and a negative relationship between these two molecules was observed in low grade glioma tissues $\left(R^{2}=0.29, p<0.001\right)$ (Figure $\left.5 D\right)$ and high grade glioma tissues $\left(\mathrm{R}^{2}=0.33, \mathrm{p}<0.001\right)$ (Figure $5 \mathrm{E}$ ). Moreover, after transfection of miR-1224-3p mimics and inhibitor in glioma cells, we examined the level of 
PLK1. We verified that the expression of PLK1 decreased both at mRNA and protein level after overexpressing miR-1224-3p, while suppressing miR1224-3p could significantly elevate the expression level of PLK1 (Figure 5F, G). Overall, we concluded that circ-ZNF609 might participate in the progression of glioma through sponging miR-1224-3p to increase the expression level of PLK1.

\section{MiR-1224-3p might inhibit proliferation, migration and invasion of glioma cells through targeting PLK1}

Next, we co-transfected glioma cells with PLK1 siRNA and miR-1224-3p inhibitor, and examined the expression level of PLK1. The results revealed that the level of PLK1 in the si-PLK1 group was remarkedly decreased, while the expression level of PLK1 in the si-PLK1+miR-1224-3p inhibitor group were obviously higher than that in the si-PLK1 group (Figure 6A). Then, we conducted in vitro experiments to explore the influence of PLK1 and miR-1224-3p on glioma cell. As shown in Figure 6B-D, we identified that decreased expression level of PLK1 in glioma cells obviously inhibited cell proliferation, migration, and invasion, while co-transfection of PLK1 siRNA and miR-1224-3p inhibitor in glioma cells respectively reversed the inhibition influence of si-PLK1 on glioma cells. This suggested that miR-1224-3p may inhibit proliferation, migration and invasion of glioma cells by targeting PLK1.
A

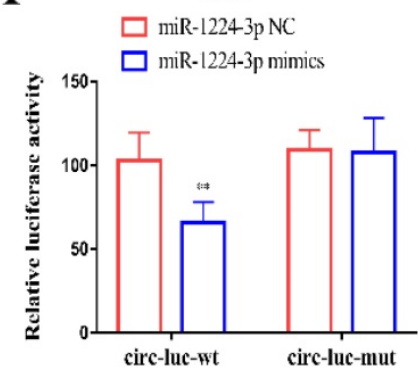

$\mathrm{C}$

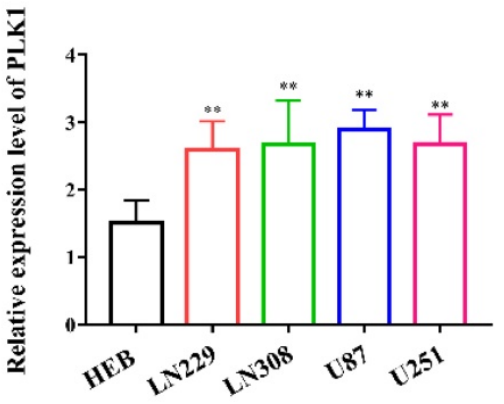

F

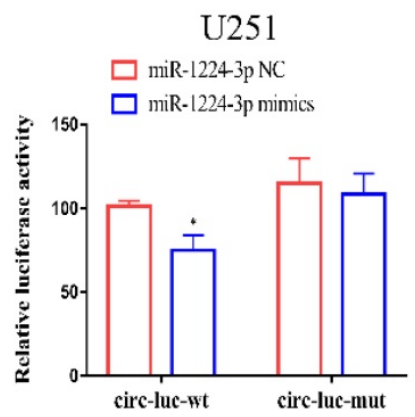

$\mathrm{D}$

$\mathrm{B}$
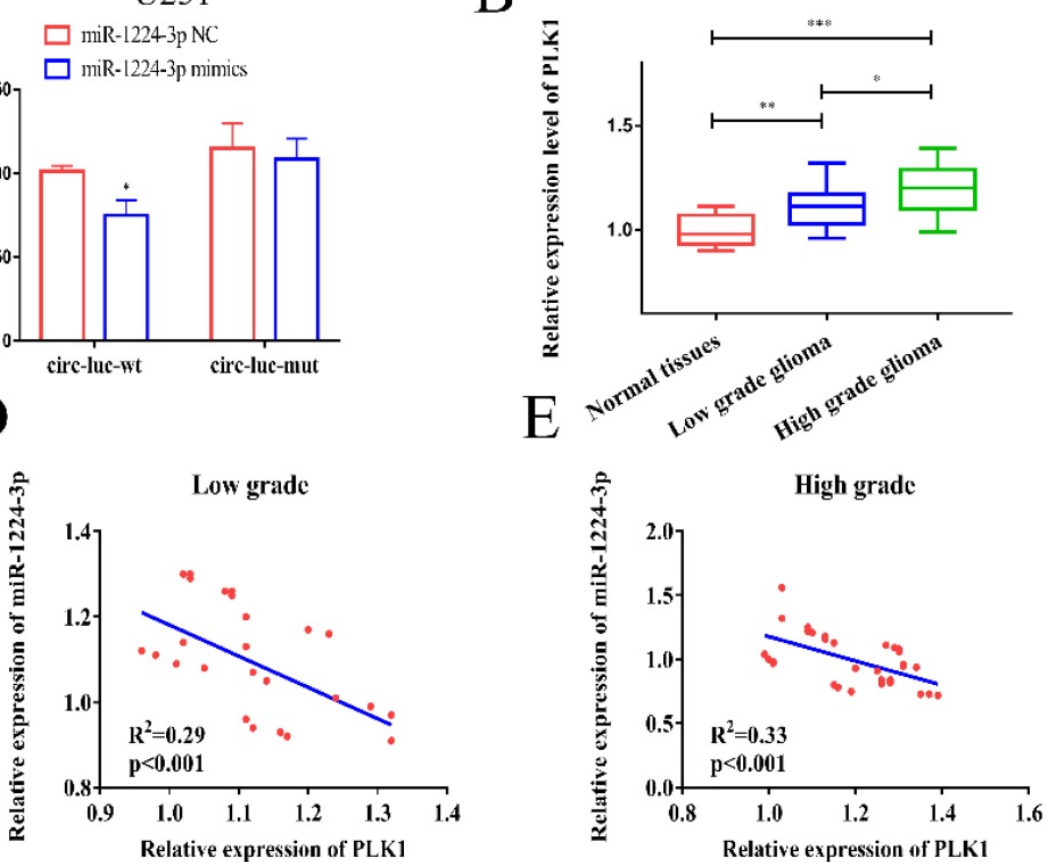

G

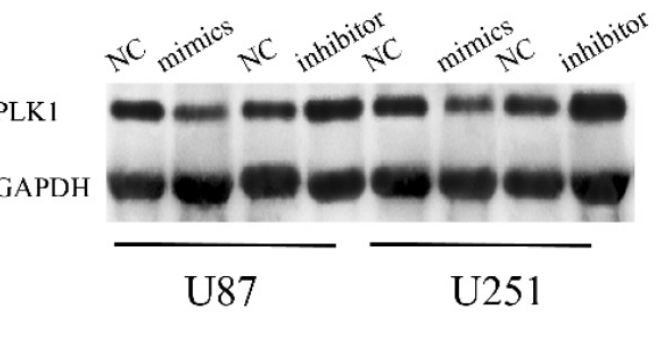

Figure 5. PLK1 is a target of miR-1224-3p. (A) Relative luciferase activities were investigated in glioma cells after transfection with PLK1-WT or PLK1-Mut and miR-1224-3p mimic or miR-NC. (B) The expression level of PLK1 in glioma tissues was increased compared to expression in matched normal tissues. (C) Relative expression of $P L K 1$ in glioma cell lines compared to expression in HEB cells. (D-E) Through Person's correlation analysis, it was found that the expression of $P L K 1$ in low grade $\left(R^{2}=0.29\right.$, $\mathrm{p}<0.001)$ and high grade glioma tissue $\left(\mathrm{R}^{2}=0.33, \mathrm{p}<0.001\right)$ was significantly negatively correlated with miR-1224-3p expression. (F-G) The mRNA and protein expression of $P L K 1$ was detected after overexpression or inhibition of miR-1224-3p in U87 and U251 cells. ${ }^{*} p<0.05 ;{ }^{* *} p<0.01 ;{ }^{* * *} p<0.001 ;{ }^{*} p<0.05 ;{ }^{*} p<0.01$. All data are showed as mean \pm SD. The experiment was conducted for three times. 
A U87

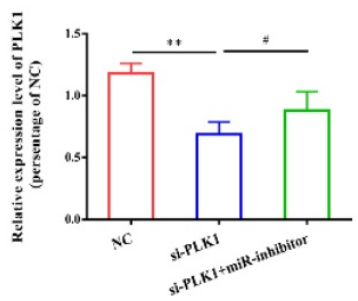

$\mathrm{C}$

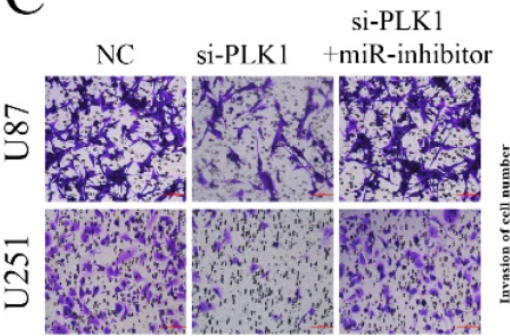

U251
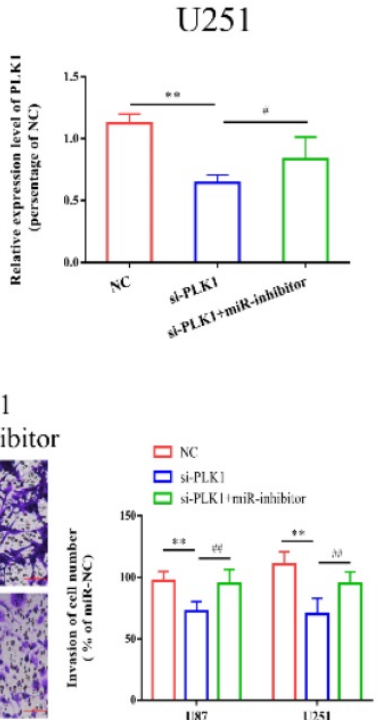

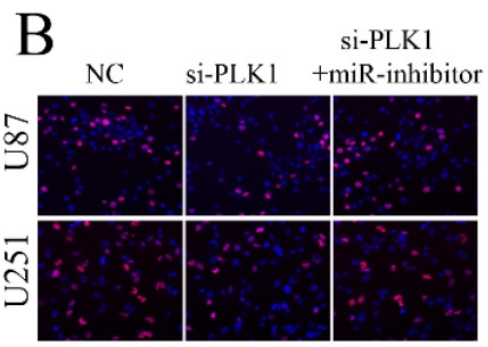

EdU/Hoechst

D

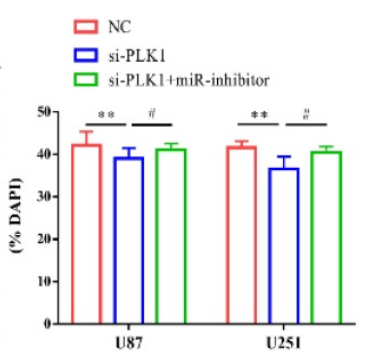

si-PLK 1

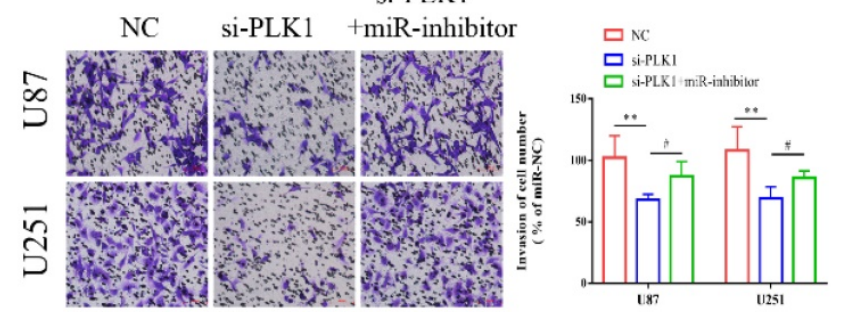

Figure 6. MiR-1224-3p might inhibit proliferation, migration and invasion of glioma cells through targeting PLK1. (A) PLK1-si and miR-1224-3p inhibitor were transfected into U87 and U251 cells, and the expression of PLK1 was measured. (B) After transfection of PLK1-si and miR-1224-3p inhibitor, EdU assay was performed to measure the cell proliferation of U87 and U251 cells (magnification: 200x). (C-D) After transfection of PLK1-si and miR-1224-3p inhibitor, Transwell assay was performed to measure the cell migration and invasion of U87 and U251 cells (magnification: 200x). ${ }^{*} *_{p}<0.01 ;{ }_{p}<0.05 ;{ }^{\prime} p<0.01$. All data are showed as mean \pm SD. The experiment was conducted for three times.

A

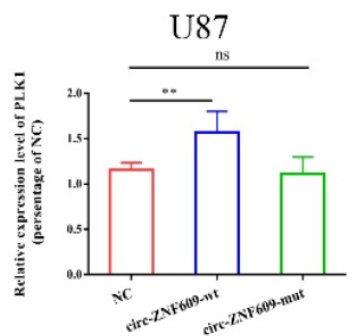

$\mathrm{B}$

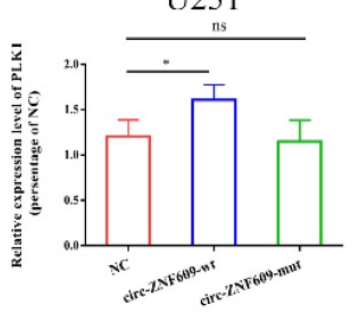

D
U251

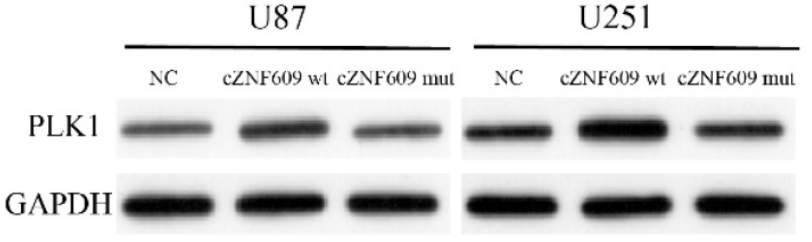

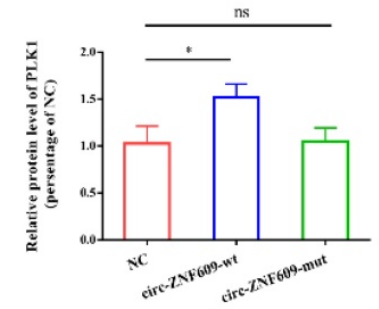

C

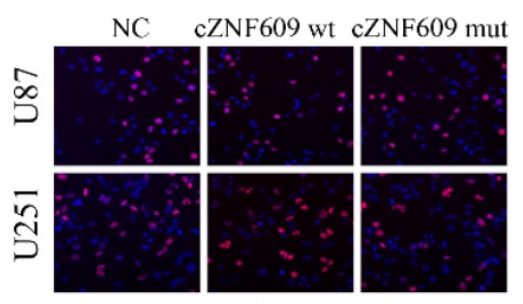

EdU/Hoechst
ㄴ. 마다.ZNF609-wt

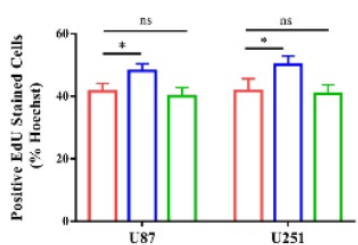

D

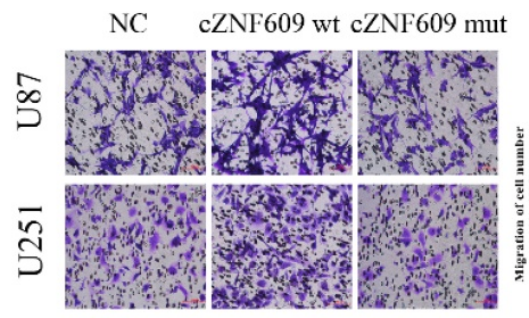

口NC 므 dic 2 NF 609 - w

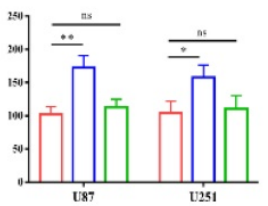

E

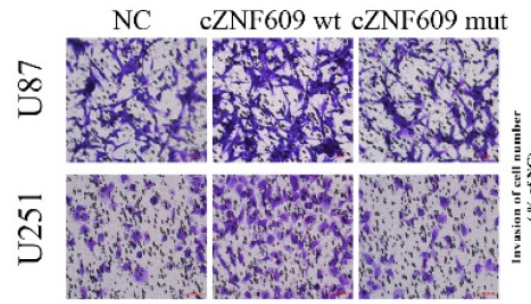

ㅁ.

당-7NF609--xt 므 cire-ZNF 609 -mu

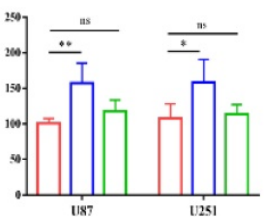

Figure 7. Circ-ZNF609 might regulate PLK1 expression by binding to miR-1 224-3p. (A-B) circ-ZNF609-wt and circ-ZNF609-mut were transfected into U87 and U251 cells, and the mRNA and protein expression level of PLK1 was measured by qRT-PCR and western blot. (C) After transfection of circ-ZNF609-wt and circ-ZNF609-mut, EdU assay was performed to measure the cell proliferation of U87 and U251 cells, the results showed that circ-ZNF609-wt could promote the proliferation of glioma cells while circ-ZNF609-mut could not affect the proliferation of cells (magnification: 200x). (D-E) After transfection of circ-ZNF609-wt and circ-ZNF609-mut, Transwell assay was performed to measure the cell migration and invasion of U87 and U251 cells, the results showed that circ-ZNF609-wt could promote the migration and invasion of glioma cells while circ-ZNF609-mut could not affect the proliferation of cells (magnification: $200 x$ ); ${ }^{*} p<0.05 ; * * p<0.01$; ns, no significant difference. All data are showed as mean \pm SD. The experiment was conducted for three times. 


\section{Circ-ZNF609 might regulate PLK1 expression by binding to miR-1224-3p}

After transfection of U87 and U251 cells with circ-ZNF609 wild type plasmid (circ-ZNF609-wt) and circ-ZNF609 mutant type plasmid (circ-ZNF609-mut), we examined the mRNA and protein expression level of PLK1 and found that PLK1 in the circ-ZNF609-wt group was remarkedly increased, while the no obvious change was observed in the circ-ZNF609-mut group (Figure 7A, B). Then, we conducted experiments to explore the effects of circ-ZNF609-wt and circ-ZNF609-mut on glioma cell. As shown in Figure 7C-E, we found that transfection of circ-ZNF609-wt in glioma cells obviously promoted cell proliferation, migration, and invasion, while transfection of circ-ZNF609-mut could not affect the cell function of glioma cells. These results suggested that circ-ZNF609 might regulate PLK1 expression through sponging miR-1224-3p.

\section{Upregulation of circ-ZNF609 promoted the growth of glioma in vivo}

We constructed stably overexpressed circ-ZNF609 or LV-NC U87 cells for the formation of tumors in immunodeficient mice to further confirm the function of circ-ZNF609 in vivo. As shown in Figure 8A-C, overexpression of circ-ZNF609 contributed to increases in tumor volume and weight in vivo. Furthermore, the results of qRT-PCR revealed that circ-ZNF609 and PLK1 expression were increased in the tumor tissues of the LV-circ-ZNF609 group, while miR-1224-3p expression was significantly decreased (Figure 8D-G). Overall, elevated expression of circ-ZNF609 markedly promoted glioma growth in vivo.
A

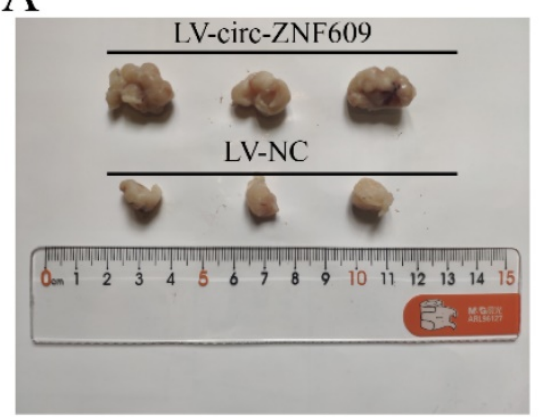

$\mathrm{D}$

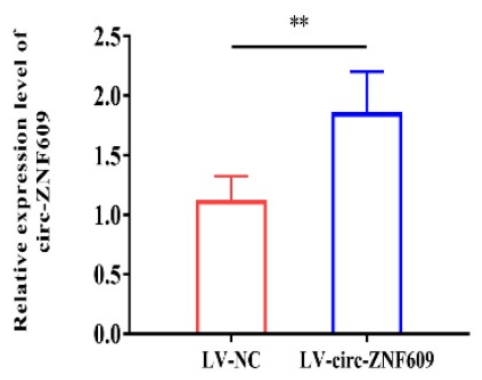

B

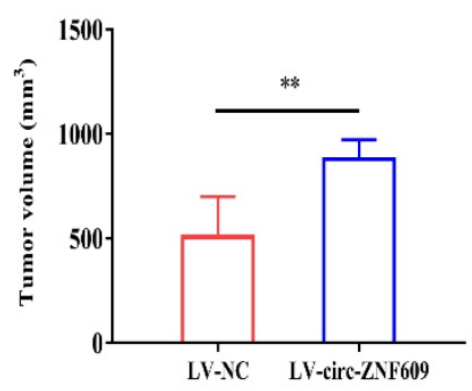

$\mathrm{E}$

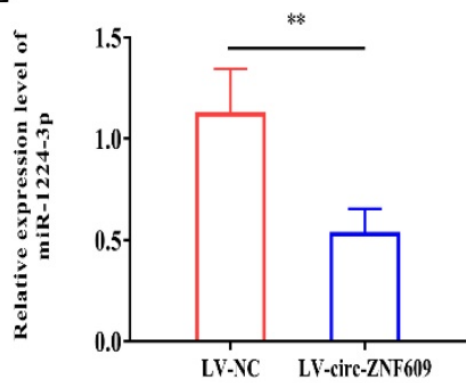

$\mathrm{C}$

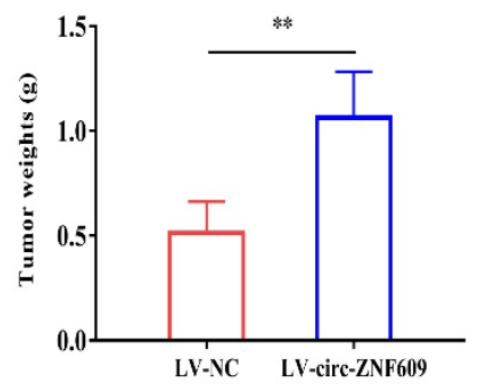

$\mathrm{F}$

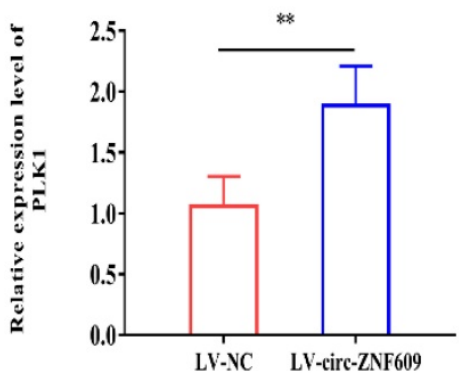

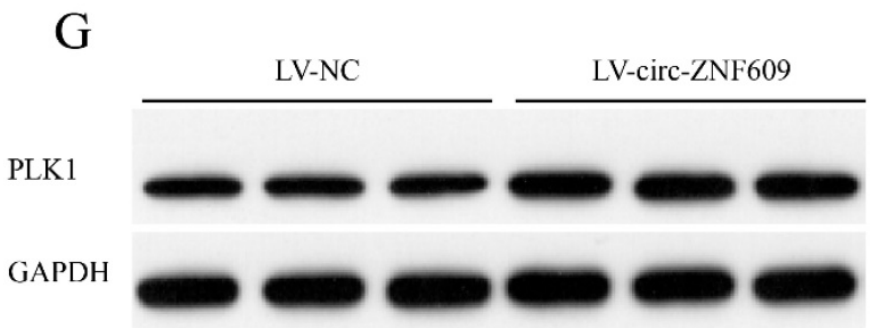

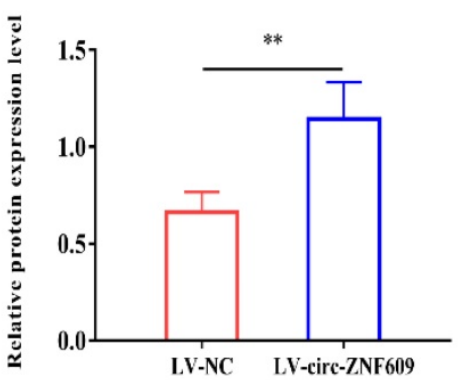

Figure 8. Upregulation of circ-ZNF609 promoted the growth of glioma in vivo. (A) Representative images of neoplasms from each group of nude mice. (B) Determination of tumor volumes. (C) Determination of tumor weights. (D) The QRT-PCR showed that circ-ZNF609 expression was incresed in tumor tissues of the LVcirc-ZNF609 group. (E) The qRT-PCR results indicated that expression of miR-1224-3p was decreased in tumor tissues of the LV-circ-ZNF609 group. F-G. QRT-PCR and western blot results indicated that circ-ZNF609 overexpression promoted PLK1 expression, $* * p<0.01$. All data are showed as mean \pm SD. The experiment was carried out for three times. 


\section{Discussion}

Glioma is a prevalent intracranial malignancy contributes to considerable cancer-related mortality $[6,27]$. Meanwhile, it is characterized by insensitive to conventional chemo-radiotherapy $[28,29]$. CircRNA is a class of noncoding RNA generated from a non-canonical back splicing process, from a covalent bond between $5^{\prime}$ and $3^{\prime}$ ends of a single-stranded RNA [30-32].

Recent years, many cancer-associated circRNAs have been identified and verified as potential clinical biomarkers and therapeutic targets in various types of tumors, including glioma. For examples, Zheng et al. found that circular RNA TTBK2 (circ-TTBK2) could promote glioma malignancy via modulating miR-217/HNF1 $\beta$ /Derlin-1 pathway [33]; Chen et al. revealed that the expression of circPTN was markedly elevated in glioma, circPTN might act as a tumour promoter through sponging miR-145-5p/miR-330-5p [34]; Cao et al. showed that hsa_circ_0037251 could function as an oncogenic gene via a hsa_circ_0037251/ miR-1224-3p/mTOR axis in glioma, and these potential biomarkers might be therapeutic targets [35]. However, the precise roles of circRNAs in human diseases remain to be further explored.

Firstly, we identified that circ-ZNF609 expression was significantly elevated in glioma tissues and cell lines by qRT-PCR assay. Besides, functional assays demonstrated that overexpression of circ-ZNF609 could markedly promote the proliferation, migration and invasion of glioma cells. Emerging evidence revealed that circRNAs might act as a ceRNA to modulate the expression of downstream genes via functioning as miRNA sponge on the whole progression of various types of diseases. As is known to us, miRNAs have the ability to serve as independent regulators in various types of biological progression. To explore the potential mechanism of circ-ZNF609, nuclear-cytoplasmic separation assay was performed and we found that circ-ZNF609 mainly existed in the cytoplasm of glioma cells, which meant that circ-ZNF609 might serve as a ceRNA to bind to miRNA, so as to regulate the expression of downstream genes. Then, we found that several miRNAs might bind to circ-ZNF609. On basis of biological function and bound fraction, we speculated that miR-1224-3p might be a potential binding target. MiR-1224-3p has been reported to be downregulated in lung adenocarcinoma and could suppress cell growth and metastasis [21]. Subsequently, the results of functional experiments demonstrated that circ-ZNF609 might sponge miR1224-3p and inhibit its expression. Besides, miR-1224$3 p$ could significantly inhibit the growth and metastasis of glioma cells. MiR-1224-3p expression was also decreased in glioma.

It has been identified that miRNAs might modulate gene expression via targeting downstream genes 3'UTR. Based on bioinformatics analysis, we found that PLK1 might be the potential target of miR-1224-3p. Polo-like kinases (PLKs) are a family of serine/threonine protein kinases which are widespread in eukaryotic cells, while PLK1 was the most investigated [36, 37]. PLK1 plays multiple roles in the biological progression, including cell division, cell cycle, cell growth and metastasis [38, 39]. PLK1 has been verified to be involved in various cancers, such as hepatocellular carcinoma [40], breast cancer [41], prostate cancer [42], acute myeloid leukemia [43]. Additionally, PLK1 has been reported to be upregulated in glioma and can markedly promote cell proliferation and migration [44-46]. Moreover, we demonstrated that circ-ZNF609 could promote the expression of PLK1 via sponge miR-1224-3p. Functional experiments identified that overexpression of PLK1 promoted the cell proliferation, migration and invasion in glioma.

In our findings, we suggest that circ-ZNF609 might significantly promote the proliferation, migration and invasion of glioma cells through the miR-1224-3p/PLK1 axis, whose function might involve molecular targets beneficial for diagnosing and treating glioma. Meanwhile, there are several parts remain to be explored in the further study. First, we need to conduct more experiments to verify other biological effects of circ-ZNF609. Second, the other potential regulatory effect of circ-ZNF609 on related pathway proteins remain to be further explored.

\section{Conclusion}

In conclusion, our study suggests that circ-ZNF609 might act as an oncogene to promote glioma progression via regulating miR-1224-3p/PLK1 axis. Therefore, circ-ZNF609 might potentially be applied in the future diagnosis and treatment in glioma.

\section{Supplementary Material}

Supplementary figure S1.

http://www.jcancer.org/v12p3354s1.pdf

\section{Acknowledgements}

This work was supported by the grant from the Jiangsu Government Scholarship for Overseas Studies. We thank Professor Kaijun Di from the department of neurology, University of California, Irvine, for her guidance with experimental methods. 


\section{Authors' contributions}

SJD and JT designed the study. SJD, FL and SZ performed the experiments; HYL and JT analyzed the data; SJD and JT wrote the paper.

\section{Data availability statement}

All data included in this study are available upon request by contacting the corresponding author.

\section{Competing Interests}

The authors have declared that no competing interest exists.

\section{References}

1. De Boeck A, Ahn BY, D'Mello C, Lun X, Menon SV, Alshehri MM, et al. Glioma-derived IL-33 orchestrates an inflammatory brain tumor microenvironment that accelerates glioma progression. Nat Commun. 2020; 11: 4997.

2. Zhang Y, Xiao Y, Li GC, Gong FY, Zhang XN, Hou K. Long non-coding RNAs as epigenetic mediator and predictor of glioma progression, invasiveness, and prognosis. Semin Cancer Biol. 2020.

3. Lou J, Hao Y, Lin K, Lyu Y, Chen M, Wang H, et al. Circular RNA CDR1as disrupts the p53/MDM2 complex to inhibit Gliomagenesis. Mol Cancer. 2020; 19: 138

4. Sa JK, Chang N, Lee HW, Cho HJ, Ceccarelli M, Cerulo L, et al. Transcriptional regulatory networks of tumor-associated macrophages that drive malignancy in mesenchymal glioblastoma. Genome Biol. 2020; 21: 216.

5. Eyler CE, Matsunaga H, Hovestadt V, Vantine SJ, van Galen P, Bernstein $B E$. Single-cell lineage analysis reveals genetic and epigenetic interplay in glioblastoma drug resistance. Genome Biol. 2020; 21: 174

6. Thakur A, Qiu G, Xu C, Han X, Yang T, Ng SP, et al. Label-free sensing of exosomal MCT1 and CD147 for tracking metabolic reprogramming and malignant progression in glioma. Sci Adv. 2020; 6: eaaz6119.

7. Azizi AA, Walker DA, Liu JF, Sehested A, Jaspan T, Pemp B, et al. NF1 optic pathway glioma. Analysing risk factors for visual outcome and indications to treat. Neuro Oncol. 2020.

8. Li F, Yang Q, He AT, Yang BB. Circular RNAs in cancer: limitations in functional studies and diagnostic potential. Semin Cancer Biol. 2020.

9. Ho-Xuan H, Glazar P, Latini C, Heizler K, Haase J, Hett R, et al. Comprehensive analysis of translation from overexpressed circular RNAs reveals pervasive translation from linear transcripts. Nucleic Acids Res. 2020; 48: 10368-82.

10. Xu X, Zhang J, Tian Y, Gao Y, Dong X, Chen W, et al. CircRNA inhibits DNA damage repair by interacting with host gene. Mol Cancer. 2020; 19: 128.

11. Gokool A, Loy CT, Halliday GM, Voineagu I. Circular RNAs: The Brain Transcriptome Comes Full Circle. Trends Neurosci. 2020; 43: 752-66.

12. Xu T, Wang M, Jiang L, Ma L, Wan L, Chen Q, et al. CircRNAs in anticancer drug resistance: recent advances and future potential. Mol Cancer. 2020; 19: 127

13. Jahng JWS, Liu L, Wu JC. Tumor Repressor Circular RNA as a New Target for Preventative Gene Therapy Against Doxorubicin-Induced Cardiotoxicity. Circ Res. 2020; 127: 483-5.

14. Ma S, Kong S, Wang F, Ju S. CircRNAs: biogenesis, functions, and role in drug-resistant Tumours. Mol Cancer. 2020; 19: 119.

15. Ding J, Wang Q, Guo N, Wang H, Chen H, Ni G, et al. CircRNA circ_0072995 promotes the progression of epithelial ovarian cancer by modulating miR-147a/CDK6 axis. Aging (Albany NY). 2020; 12: 17209-23.

16. Guo J, Su Y, Zhang M. Circ_0000140 restrains the proliferation, metastasis and glycolysis metabolism of oral squamous cell carcinoma through upregulating CDC73 via sponging miR-182-5p. Cancer Cell Int. 2020; 20: 407.

17. Wang Y, Yin L, Sun X. CircRNA hsa_circ_0002577 accelerates endometrial cancer progression through activating IGF1R/PI3K/Akt pathway. J Exp Clin Cancer Res. 2020; 39: 169.

18. Rossi F, Legnini I, Megiorni F, Colantoni A, Santini T, Morlando M, et al. Circ-ZNF609 regulates G1-S progression in rhabdomyosarcoma. Oncogene. 2019; 38: 3843-54

19. Laity JH, Lee BM, Wright PE. Zinc finger proteins: new insights into structural and functional diversity. Curr Opin Struct Biol. 2001; 11: 39-46.
20. Xiong $\mathrm{Y}$, Zhang J, Song C. CircRNA ZNF609 functions as a competitive endogenous RNA to regulate FOXP4 expression by sponging miR-138-5p in renal carcinoma. J Cell Physiol. 2019; 234: 10646-54.

21. Zuo Y, Shen W, Wang C, Niu N, Pu J. Circular RNA Circ-ZNF609 Promotes Lung Adenocarcinoma Proliferation by Modulating miR-1224-3p/ETV1 Signaling. Cancer Manag Res. 2020; 12: 2471-9.

22. Zhu L, Liu Y, Yang Y, Mao XM, Yin ZD. CircRNA ZNF609 promotes growth and metastasis of nasopharyngeal carcinoma by competing with microRNA-150-5p. Eur Rev Med Pharmacol Sci. 2019; 23: 2817-26.

23. Wu L, Xia J, Yang J, Shi Y, Xia H, Xiang X, et al. Circ-ZNF609 promotes migration of colorectal cancer by inhibiting Gli1 expression via microRNA-150. J BUON. 2018; 23: 1343-9.

24. Liao X, Zhan W, Tian B, Luo Y, Gu F, Li R. Circular RNA ZNF609 Promoted Hepatocellular Carcinoma Progression by Upregulating PAP2C Expression via Sponging miR-342-3p. Onco Targets Ther. 2020; 13: 7773-83

25. Liu Z, Pan HM, Xin L, Zhang Y, Zhang WM, Cao P, et al. Circ-ZNF609 promotes carcinogenesis of gastric cancer cells by inhibiting miRNA-145-5p expression. Eur Rev Med Pharmacol Sci. 2019; 23: 9411-7.

26. Du S, Zhang P, Ren W, Yang F, Du C. Circ-ZNF609 Accelerates the Radioresistance of Prostate Cancer Cells by Promoting the Glycolytic Metabolism Through miR-501-3p/HK2 Axis. Cancer Manag Res. 2020; 12: 7487-99.

27. Tao W, Chu C, Zhou W, Huang Z, Zhai K, Fang X, et al. Dual Role of WISP1 in maintaining glioma stem cells and tumor-supportive macrophages in glioblastoma. Nat Commun. 2020; 11: 3015.

28. Pattwell SS, Arora S, Cimino PJ, Ozawa T, Szulzewsky F, Hoellerbauer P, et al. A kinase-deficient NTRK2 splice variant predominates in glioma and amplifies several oncogenic signaling pathways. Nat Commun. 2020; 11: 2977.

29. Liu SJ, Malatesta M, Lien BV, Saha P, Thombare SS, Hong SJ, et al. CRISPRi-based radiation modifier screen identifies long non-coding RNA therapeutic targets in glioma. Genome Biol. 2020; 21: 83.

30. Wu Y, Zhang Y, Zheng X, Dai F, Lu Y, Dai L, et al. Circular RNA circCORO1C promotes laryngeal squamous cell carcinoma progression by modulating the let-7c-5p/PBX3 axis. Mol Cancer. 2020; 19: 99.

31. Huang XY, Zhang PF, Wei CY, Peng R, Lu JC, Gao C, et al. Circular RNA circMET drives immunosuppression and anti-PD1 therapy resistance in hepatocellular carcinoma via the miR-30-5p/snail/DPP4 axis. Mol Cancer. 2020; 19: 92.

32. Han D, Wang Y, Wang Y, Dai X, Zhou T, Chen J, et al. The Tumor-Suppressive Human Circular RNA CircITCH Sponges miR-330-5p to Ameliorate Doxorubicin-Induced Cardiotoxicity Through Upregulating SIRT6, Survivin, and SERCA2a. Circ Res. 2020; 127: e108-e25.

33. Zheng J, Liu X, Xue Y, Gong W, Ma J, Xi Z, et al. TTBK2 circular RNA promotes glioma malignancy by regulating miR-217/HNF1beta/Derlin-1 pathway. J Hematol Oncol. 2017; 10: 52.

34. Chen J, Chen T, Zhu Y, Li Y, Zhang Y, Wang Y, et al. circPTN sponges miR-145-5p/miR-330-5p to promote proliferation and stemness in glioma. J Exp Clin Cancer Res. 2019; 38: 398

35. Cao Q, Shi Y, Wang X, Yang J, Mi Y, Zhai G, et al. Circular METRN RNA hsa_circ_0037251 Promotes Glioma Progression by Sponging miR-1229-3p and Regulating mTOR Expression. Sci Rep. 2019; 9: 19791.

36. Liu Z, Sun Q, Wang X. PLK1, A Potential Target for Cancer Therapy. Transl Oncol. 2017; 10: 22-32

37. Gutteridge RE, Ndiaye MA, Liu X, Ahmad N. Plk1 Inhibitors in Cancer Therapy: From Laboratory to Clinics. Mol Cancer Ther. 2016; 15: 1427-35.

38. Combes G, Alharbi I, Braga LG, Elowe S. Playing polo during mitosis: PLK1 takes the lead. Oncogene. 2017; 36: 4819-27.

39. Ruf S, Heberle AM, Langelaar-Makkinje M, Gelino S, Wilkinson D, Gerbeth C, et al. PLK1 (polo like kinase 1) inhibits MTOR complex 1 and promotes autophagy. Autophagy. 2017; 13: 486-505

40. Li Y, Zhao ZG, Luo Y, Cui H, Wang HY, Jia YF, et al Dual targeting of Polo-like kinase 1 and baculoviral inhibitor of apoptosis repeat-containing 5 in TP53-mutated hepatocellular carcinoma. World J Gastroenterol. 2020; 26: 4786-801.

41. Montaudon E, Nikitorowicz-Buniak J, Sourd L, Morisset L, El Botty R, Huguet L, et al. PLK1 inhibition exhibits strong anti-tumoral activity in CCND1-driven breast cancer metastases with acquired palbociclib resistance. Nat Commun. 2020; 11: 4053.

42. Gheghiani L, Shang S, Fu Z. Targeting the PLK1-FOXO1 pathway as a novel therapeutic approach for treating advanced prostate cancer. Sci Rep. 2020; 10: 12327.

43. Guan J, Liu P, Wang A, Wang B. Long noncoding RNA ZEB2AS1 affects cell proliferation and apoptosis via the miR1225p/PLK1 axis in acute myeloid leukemia. Int J Mol Med. 2020; 46: 1490-500.

44. Zhang R, Wei RL, Du W, Zhang LW, Du T, Geng YD, et al. Long noncoding RNA ENST00000413528 sponges microRNA-593-5p to 
modulate human glioma growth via polo-like kinase 1 . CNS Neurosci Ther. 2019; 25: 842-54.

45. Wu ZY, Wei N. Knockdown of PLK1 inhibits invasion and promotes apoptosis in glioma cells through regulating autophagy. Eur Rev Med Pharmacol Sci. 2018; 22: 2723-33.

46. Amani V, Prince EW, Alimova I, Balakrishnan I, Birks D, Donson AM, et al. Polo-like Kinase 1 as a potential therapeutic target in Diffuse Intrinsic Pontine Glioma. BMC Cancer. 2016; 16: 647. 